\title{
Kochi Core Center Provides New Information Services
}

\author{
by Lallan Gupta, Toshio Hisamitsu, and Noriaki Masui
}

\section{Introduction}

During the last four decades of scientific drilling of seafloor all over the world, an enormous amount $(2360,000 \mathrm{~m})$ of marine cores has been collected. These cores are being curated in the three Integrated Ocean Drilling Program (IODP) core repositories located in Germany, Japan, and the U.S.A.. The IODP core repository in Japan is housed in the Kochi Core Center (KCC), Kochi on the island of Shikoku. The total amount of IODP core currently being curated in the KCC is about 93,000 m. These cores were collected from the Indian and west Pacific Oceans, and they consist of a diverse range of geological material. Detailed description of the core material is available in preliminary reports of each drilling expedition. This report details three new services being offered through the KCC website (www.kochi-core. jp): Core Summary, Routine Microbiological Sample (RMS), and Virtual Core Library (VCL).

\section{Core Summary}

Cores contain a wide variety of material for the earth science community. Although much of the research is carried out by taking bulk samples from cores, some researchers have specific interests for subsamples, such as ash, calcite nodules, or plankton fossils. However, such detail was so far only provided in individual volumes of preliminary reports or proceedings of expeditions of the IODP core material. For an electronic access to these data, the KCC curatorial staff compiled major features of sample material recovered through Deep Sea Drilling Project (DSDP), Ocean Drilling Program (ODP) and IODP, and made the "Core Summary" information publically available through the KCC web page (http://www.kochi-core.jp/cs/).

The core summary web page lists basic parameters like expedition or leg number, site number, hole, geographical location of the drilled site, drilled interval depth, total length of recovered core, approximate geological age range covered by the core sediments, an overview of lithology, age model, and magnetostratigraphy, together with the link for the preliminary report or proceedings of each drilling expedition. The list can be filtered using various combinations of keywords and parameters. This action will facilitate short-listing of the information related to the core material, which is dispersed in the individual volumes of the preliminary report or proceedings of each leg/expedition. For quickly locating the drill site, a click on the site name takes the user to geographical map services with the site clearly marked on it. Lithological summary of core material recovered from each hole can be quickly viewed in a pop-up window or a new window. Age model, core recovery, and magnetostratigraphy information extracted from preliminary reports or proceedings of each leg/expedition are available in PDF format, which can easily be downloaded.

\section{Routine Microbiological Samples (RMS)}

Subsequent to the discovery of life and biosphere in subseafloor environments, the study of biomass, activity, and function of subseafloor life and its ecological roles in biogeochemical cycles has become one of the major scientific objectives in the IODP. In addition, biochemical analytical techniques based on DNA are rapidly developing. Therefore, IODP's Science Advisory Structure (SAS) recommended routine sampling for geomicrobiology, and core repositories were asked to develop the capabilities for storage of core samples at very low temperature. The Japanese Center for Deep Earth Exploration (CDEX) initiated routine collection of microbiological sub-samples of cores in 2009, and these samples are being curated in the KCC.

Storage of the RMS under deep-freezing conditions is critical for preventing degradation-caused by abiotic hydrolysis, enzymatic reactions, and possible contamination-of fragile bio-molecules such as DNA, RNA, enzymes, sugar chains, and intact polar lipids. Moreover, aseptic sub-sampling and distribution of the RMS without thawing is desired for quality assurance purposes. KCC is equipped with deep freezers $\left(-80^{\circ} \mathrm{C}\right)$ and liquid nitrogen-cooled tanks $\left(-160^{\circ} \mathrm{C}\right)$ for long-term storage and with an aseptic band saw system for "clean" processing of the frozen core sample (Masui et al., 2009). Using these geobiological sampling devices, aliquots of the RMS can be distributed to the science community worldwide according to IODP policy. To spread information about IODP and RMS, KCC prepared a leaflet and a handbill for distribution at academic meetings. In addition, an RMS promotion site has been added to the $\mathrm{KCC}$ website. Overview of RMS curation and a list of the RMS have been made available through this website. The list can be filtered for sample depth, expedition, and site name in order to find suitable RMS. 


\section{Virtual Core Library (VCL)}

The drilling vessel Chikyu is equipped with an X-ray Computed Tomography (XCT) scanner, which provides the capability to visualize the internal structure of cores to aid in discerning important geological features prior to splitting and discrete sampling of the cored materials. Standard core flow on board Chikyu involves XCT scanning of cores before they are split and archiving of the digital core images (Fig. 1). These data are used by shipboard scientists to identify key features within a core section and to decide if whole round core (WRC) sampling and preservation of part of an unsplit core is necessary for future investigations. The XCT scanning of one core section produces a file that contains data for previewing a 2-D image and for reconstructing a 3-D image of the core section. The files are saved into a format known as DICOM (Digital Imaging and Communication in Medicine). The J-CORES data server stores all files related to the XCT-scanned images, and provides access to the files via the Internet. Files are freely available after the expedition moratorium period.

KCC designed a new web page named "Virtual Core Library" (VCL) that introduces and promotes use of the XCT data for researchers and educators, and displays digital 3-D images of typical core sections on the gallery page. Users can also access preliminary reports of Chikyu expeditions and understand scientific details related to a particular cruise, site, hole, etc. The VCL provides a link to the coronal images (2-D images) of core sections that can be quickly viewed. Researchers can then request a copy of DICOM files

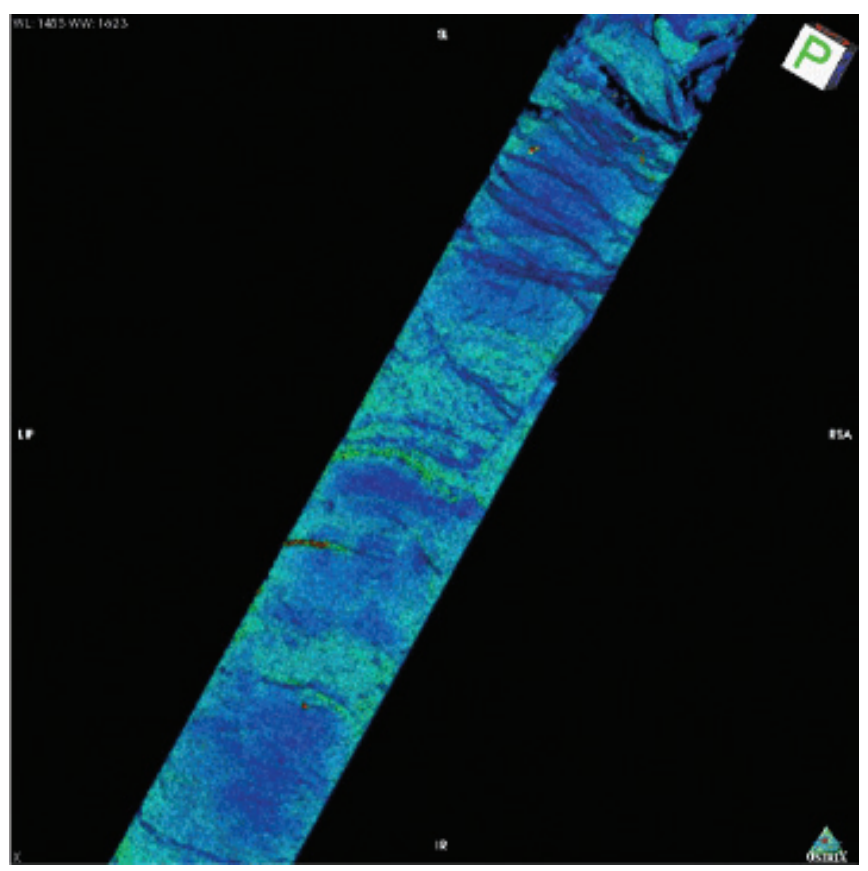

Figure 1. The OsiriX-rendered false colored image of a core section based on XCT scanning data. Fractures and gaps are easily visualized in this image. Change in color from light blue to red can be interpreted to be a reflection of change in density of core material, although calibration is a must to conclude about density. for core sections of interest. After examining details of the request, curatorial staff will provide the data either by copying them on a DVD or through a file transfer protocol (FTP) setup.

Recently, DICOM viewing software has become freely available on the Internet (e.g., OsiriX, ApolloView Lite, Onis Viewer). Using such software, researchers can render 3-D images of core sections from DICOM files and can visualize the internal structure of cores by changing transparency and color and by rotating or slicing the image. General DICOM viewers have functions like rendering, cutting, or slicing 3-D images, and they provide an opportunity for users to "virtually sample". The VCL will be improved to accelerate use of the XCT data and promote new approaches in geological studies.

The KCC curatorial staff will continue to improve these Internet-based services to allow for the best possible utilization of IODP samples by researchers around the world, and thus contribute in enhancing our current understanding of the Earth and its environments.

\section{References}

Masui, N., Morono, Y., and Inagaki, F., 2009. Bio-Archive core storage and subsampling procedure for subseafloor molecular biological research. Sci. Drill., 8:35-37. doi:10.2204/iodp. sd.8.05.2009

\section{Authors}

Lallan Gupta, Toshio Hisamitsu, and Noriaki Masui, Kochi Core Center, 200 Monobe Otsu, Nankoku, Kochi 783-8502, Japan, e-mail: gupta@jamstec.go.jp.

\section{Related Web Links}

http://www.kochi-core.jp/cs/

http://www.kochi-core.jp/rms/

http://www.kochi-core.jp/VCL/ 